\title{
GLAD!
}

Revue sur le langage, le genre, les sexualités

11 | 2021

Archives, genre, sexualités, discours

\section{Faire parler le « rien »}

À la recherche du corps et de la sexualité dans les archives

Making Absence Speaks. In Search of the Body and Sexuality in the Archives

\section{Marine Gilis}

\section{(2) OpenEdition}

\section{Journals}

Édition électronique

URL : https://journals.openedition.org/glad/2984

ISSN : 2551-0819

\section{Éditeur}

Association GSL

\section{Référence électronique}

Marine Gilis, « Faire parler le « rien » », GLAD! [En ligne], 11 | 2021, mis en ligne le 20 décembre 2021 , consulté le 29 janvier 2022. URL : http://journals.openedition.org/glad/2984

Ce document a été généré automatiquement le 29 janvier 2022.

\section{(c) $(1) \odot$}

La revue GLAD! est mise à disposition selon les termes de la Licence Creative Commons Attribution -

Pas d'Utilisation Commerciale - Pas de Modification 4.0 International. 


\title{
Faire parler le « rien »
}

\author{
À la recherche du corps et de la sexualité dans les archives \\ Making Absence Speaks. In Search of the Body and Sexuality in the Archives
}

\section{Marine Gilis}

1 Journal de bord, 18 juillet 2019, Brest :

Lundi, je me réveille joyeuse, c'est journée archives. J'y suis pour l'ouverture et je me précipite à la recherche de fonds. À midi, je repars bredouille. Quatre feuilles sur l'IVG en 1979, et puis rien. Simone tente de me consoler. Elle a de nombreux contacts à me donner, des personnes à me faire rencontrer. Elle passe des appels pour moi, je suis très touchée par son soutien dans mes recherches. [...]

Le dernier matin à Brest, le 17, je retourne aux archives municipales explorer les fonds sur la LCR [Ligue communiste révolutionnaire], j'avais un petit espoir d'y trouver une trace des mobilisations féministes des années 1970. Rien.

2 J'ai écrit ce texte sur mon carnet de terrain en juillet 2019. J'effectuais un voyage en itinérance à vélo pendant trois semaines, des Côtes-d'Armor au Finistère, pour visiter des centres d'archives et réaliser des entretiens afin de comprendre l'expérience de libération sexuelle des militantes du Mouvement des femmes. Dans ces notes, un mot est important, c'est le mot « rien ». Ce rien est-il vraiment rien ou est-ce moi qui ne sais pas quoi faire de ce que j'ai découvert? Ce rien a en réalité plusieurs sens. C'est d'abord le rien du peu de matière, ces quatre feuilles sur les statistiques du nombre d'IVG (interruption volontaire de grossesse) en 1979 à Brest. C'est aussi le rien de l'impersonnel alors que je travaille sur l'expérience de vie, l'intime, la voix des militantes. Mais ce n'est pas absolument rien. Ces statistiques révèlent des manières de catégoriser des expériences du corps des femmes et de leur environnement social et familial. Elles ne distinguent pas les féministes des autres et en ce sens rendent anonyme et général ce que j'interroge sur le plan de l'individualité. C'est aussi tout un discours de et sur l'institution judiciaire, policière, politique et médicale. Face à ce rien qui n'est pas tout à fait rien, il me restait à rencontrer les militantes pour constituer un quelque chose et le transmettre à d'autres. Plusieurs dizaines de témoignages ont été 
recueillis pendant ma thèse et dans le cadre de mon engagement au sein de l'association Archives du féminisme ${ }^{1}$. J'ai rejoint la commission audiovisuelle d'Archives du féminisme en 2018 après réflexion sur l'accessibilité de la recherche et notamment des sources que sont les témoignages. Cet article porte ainsi sur une double démarche, militante et de recherche. Le travail de thèse que j'effectue au sein de l'Université d'Angers et du laboratoire TEMOS a pour intitulé «L'expérience de libération sexuelle des militantes du Mouvement des femmes en Bretagne et Pays de la Loire (1970-1981)». Les militantes que je rencontre dans le cadre de cette recherche sont celles que je filme pour la constitution d'un fonds d'archives orales au sein d'Archives du féminisme.

3 À partir d'un travail sur l'expérience sexuelle et amoureuse d'une catégorie de personnes, comment trouver et exploiter les archives existantes? Que faire en cas d'absence de ces archives? Je commencerai par une présentation des lieux qui conservent les archives relatives aux luttes féministes dans les années 1970 dans l'ouest de la France métropolitaine et une analyse des fonds existants. J'évoquerai ensuite la recherche des mots pour appréhender le corps et la sexualité dans les sources écrites. Je terminerai sur la constitution d'un fonds d'archives orales comme moyen de pallier un manque de sources écrites.

\section{Pas d'archives, pas d'histoire}

4 Les archives sont au centre du travail de l'historien.ne. D'abord examinées sous l'angle de l'histoire de l'archivistique ou de l'institution des Archives nationales, elles font l'objet, depuis quelques années, d'un regard nouveau porté par l'anthropologie, l'histoire politique et sociale, mais aussi grâce à des travaux qui interrogent les lieux de l'archive. Les archives constituent désormais un objet de recherche à part entière (Artières 2009 : 119-126). Depuis quelques années émergent également des travaux de recherche sur les archives liées à l'histoire des femmes. En 2015 s'est tenue une journée d'étude aux Archives nationales de Pierrefitte-sur-Seine intitulée "Dans les coulisses des archives : où sont les femmes? ». Cette journée d'étude a soulevé des interrogations sur les pratiques archivistiques aussi bien que sur l'historiographie, en axant notamment sur le rôle des archivistes, bibliothécaires et historien.nes dans l'écriture et la valorisation de l'histoire des femmes et du genre. De la même manière, l'ouvrage paru en 2017, Le Genre de l'archive. Constitution et transmission des mémoires militantes (Blum 2017), met au centre la double question posée par Michelle Perrot, celle de l'existence et de la valorisation des sources sur les femmes. En mars 2018 s'est tenu à Angers un colloque international intitulé « Les féministes et leurs archives (1968-2018). Militantisme, mémoire et recherche ${ }^{2} »$. Deux mois plus tard, la ministre de la Culture, Françoise Nyssen, annonçait une grande collecte nationale d'archives autour de la place des femmes dans le monde du travail, répondant ainsi au rapport de Françoise Thébaud $^{3}$ qui a fait un état des lieux des pratiques en matière de collecte d'archives des femmes. Un travail de recension avait déjà été effectué : le Guide des sources de l'histoire du féminisme paru aux PUR en 2006 (Bard, Metz \& Neveu 2006) a été le fruit d'un travail collectif de l'association Archives du féminisme ${ }^{4}$. Le manque de sources a été longtemps un prétexte pour ne pas intégrer les femmes dans l'histoire. Il tient à un certain nombre de facteurs : l'invisibilité des femmes dans l'espace public, leur exclusion de la sphère politique, le fait que les femmes se dévalorisent et laissent peu de traces écrites 
et matérielles, une réticence et une sélection quand il s'agit de préserver les archives de femmes, des documents et statistiques asexués qui cachent les « elles» au profit du « il »... (Michelle Perrot 2008 : 14-18). Un tournant s'opère à partir des années 1980 avec le développement des études féministes et la multiplication des centres de documentation et d'archives spécialisés sur les femmes, les lesbiennes et le féminisme. Si la bibliothèque Marguerite Durand ${ }^{5}$ existe dès le début des années 1930, la structure qui conserve désormais le plus grand nombre de fonds a été créée en 2000, il s'agit du Centre des archives du féminisme ( $\left.\mathrm{CAF}^{6}\right)$. Un centre d'archives et de documentation lesbien, les Archives Recherches Cultures Lesbiennes (ARCL), existe également à Paris depuis 1983.

5 Soixante fonds d'archives ont été explorés dans le cadre de ma thèse, issus des Archives départementales (Côtes-d'Armor, Ille-et-Vilaine, Loire-Atlantique, Mayenne, Maine-etLoire), des Archives municipales (Brest, le Mans, Laval, Rennes, Saint-Brieuc), du Centre des archives du féminisme à Angers, de la Cinémathèque et du Musée de Bretagne et, de façon virtuelle, du Centre d'Histoire du Travail de Nantes ${ }^{7}$. Aux Archives départementales et municipales, les archives qui m'ont été utiles sont classées dans les séries W (archives publiques postérieures à 1940) et J (fonds personnels). À cela s'ajoutent quelques documents de la série FI (documents figurés) et la série JP (périodiques). Le « rien » évoqué dans l'extrait du carnet de terrain commence avec des recherches sur les inventaires en ligne des centres d'archives municipaux et départementaux. Quels mots-clés utiliser? Le mot-clé « MLF » n'a jamais rien donné, ni «Veil », ni « trotskyste » et ni « maoïste » (je pensais qu'en allant chercher du côté des groupes et partis politiques dont les militantes ont pu faire partie, j'aurais pu trouver quelques traces de luttes ou tracts féministes). Le mot "féminisme » n'a donné qu'un résultat $^{8}$ sur l'ensemble des catalogues des archives départementales et municipales. Un mot-clé sert à caractériser le contenu d'un document (personne, lieu, sujet) et permet l'indexation dans une base de données. L'invisibilité des luttes féministes tient ici au fait qu'aucun des contenus, des sujets ou des personnes n'ait été caractérisé comme «féministe » dans les bases de données. Le mot «femme(s) » est, quant à lui, largement utilisé, notamment dans la description de cartes postales de type ethnographique. Une recherche avec ce mot-clé n'est toutefois possible que dans des centres d'archives municipales, les archives départementales offrant trop de résultats. À l'inverse, le mot « homosexualité » ne donne des résultats que dans les grandes villes comme Rennes et le mot «lesbienne » n'existe pas. Des mots-clés apparaissent pour décrire de nouvelles pratiques comme "IVG» qui concerne la période postérieure à 1970. Il est utilisé en parallèle du mot «avortement » qui lui est parfois préféré selon des critères non identifiables. Le thème de la contraception est beaucoup moins présent dans les inventaires et semble avoir moins préoccupé les institutions judiciaires et policières. Il est à noter que les catalogues en ligne sont incomplets, le travail de numérisation des inventaires et d'indexation est en cours et seules une visite et une demande de conseils auprès des archivistes permettent de s'assurer de ne pas mettre de côté des fonds pertinents pour une recherche.

6 À qui et à quoi les administrations françaises s'intéressent-elles ? Issues de cabinets du préfet, les archives de la série $\mathrm{W}$ peuvent être regroupées en quelques thèmes principaux: vie associative, renseignements généraux/sécurité publique, délégation régionale aux droits des femmes et affaires sanitaires et sociales. Voici un bref aperçu de ce que peuvent contenir ces fonds W. Le fonds 1127W25 des AD des Côtes-d'Armor 
contient, entre autres, plusieurs rapports de la Direction centrale des renseignements généraux qui relatent les assises régionales du mouvement "Laissez-les-vivre » du 29 octobre 1978 à Dinan et les réactions féministes, syndicales et de la gauche qui ont suivi, notamment la rencontre régionale féministe du 2 décembre 1978. Le fonds 1772W4 des Archives départementales de la Mayenne contient des archives sur le centre d'interruption volontaire de grossesse ouvert à Laval en mars 1976 (lettres, documents administratifs sur le recrutement de médecins vacataires, statistiques, documents relatifs au protocole et matériel utilisé pour pratiquer les IVG). Le fonds 408W500 des Archives du Mans regroupe des archives relatives à la Grande Conférence du Maine sur « Contraception ou avortement? » du 9 novembre 1973, dont les invitée.e.s étaient l'avocate Gisèle Halimi et le docteur Emile Hervet. Aux AD de Loire-Atlantique. Le fonds $2059 \mathrm{~W} 2$ contient les différents statuts de l'association Centre des femmes de Nantes dont on peut voir l'évolution à partir de sa création en mars 1977. Les archives de la série $\mathrm{W}$ permettent de trouver trace d'évènements organisés par des féministes, de comprendre leur réception au niveau politique, les relations entre militantes et institutions mais aussi tous les enjeux liés à la surveillance policière, la mise en place des centres IVG et de planification ainsi que l'évolution législative par rapport aux actions et revendications féministes.

7 Les fonds personnels que j'ai consultés, ceux de la série J dans les centres d'archives départementaux et municipaux, sont des fonds déposés par des hommes, à l'exception de celui de Ghislaine Ménage qui était en cours de dépôt aux Archives municipales de Rennes et n'était pas encore visible dans les inventaires. Une autre militante m'a signalé avoir donné un fonds d'archives aux Archives de Brest mais il n'a pas été trouvé pendant ma visite. J'ai travaillé sur trois fonds personnels au volume important : celui de Philippe le Pichon (Archives départementales de Loire-Atlantique, $275 \mathrm{~J} 86$ ), de JeanJoseph Chevalier (Archives départementales d'Ille-et-Vilaine, $37 \mathrm{~J} 10$ ) et de Patrick Wiener (Archives municipales de Rennes $56 \mathrm{Z} 1$ à 12 et 33). Qui sont-ils ? Philippe le Pichon, anciennement maitre de conférences en science politique à l'Université de Nantes, a un parcours émaillé d'engagements dans des groupes et structures éclectiques qui vont de la recherche en Sciences humaines et sociales à des associations médicales, culturelles ou encore de parents d'élèves. Il a participé notamment dès 1968 à l'Assemblée générale pour l'avortement. Sa femme, Marie-Françoise le Pichon, est à peine mentionnée dans l'inventaire détaillé et pourtant, elle était très active au sein de l'AG pour l'avortement et les documents proviennent peut-être d'elle ${ }^{9}$. La présentation de Jean-Joseph Chevalier est moins fournie. L'inventaire nous apprend qu'il a déposé une collection de journaux, bulletins, tracts et documents divers concernant les mouvements étudiants, politiques, syndicaux et démocratiques de gauche et d'extrême gauche couvrant la période 1970-1976. Patrick Wiener est un médecin qui a donné des archives de l'association Choisir-Rennes: lettres, tracts, fiches de renseignement, documents pour comprendre la méthode d'aspiration dite "méthode Karman ", mais aussi des documents sur les activités du Groupe de libération homosexuelle de Rennes. La richesse de ce fonds permet de comprendre la réalité pratique de l'avortement avant sa légalisation en 1975 et celles des groupes féministes et homosexuels sur la ville. Le fonds Alain Chénard ( $\mathrm{AD}$ de Loire Atlantique, $283 \mathrm{~J}$ 122) aurait pu être intéressant, mais il n'est pas communicable avant 2038. Il comporte des documents sur le projet de reconduction définitive de la loi Veil (propositions de loi, correspondance, notes, tracts, pétitions, rapports, lettres...) entre 1976 et 1987. Alain Chénard ${ }^{10}$, ingénieur informatique puis inspecteur général des PTT et membre du Conseil général des 
Technologies de l'Information, a eu plusieurs engagements syndicaux et politiques. Il a notamment été maire de Nantes de 1977 à 1983, député socialiste de la Loire-Atlantique de 1978 à 1988 et conseiller régional de 1978 à 1986. Les engagements féministes constituent une parenthèse dans la vie de ces hommes qui ont déposé des fonds relatifs à plusieurs de leurs engagements. Les hommes sont, en effet, présents au Mouvement français pour le Planning familial, surtout à ses débuts, ainsi qu'au MLAC (Mouvement pour la liberté de l'avortement et de la contraception) et, dans une moindre mesure, à Choisir et dans des collectifs locaux de luttes pour la contraception et l'avortement comme l'Assemblée générale pour l'avortement à Nantes, le GALAC (groupe angevin pour la libéralisation de l'avortement et de la contraception) à Angers, le CRAC (Collectif rennais pour l'avortement et la contraception) à Rennes. Des hommes s'organisent également pour la promotion et la pratique de la stérilisation masculine notamment au sein d'ARDECOM (Association pour la recherche et le développement de la contraception masculine). La lutte pour l'accès à la contraception et l'avortement implique une mobilisation des médecins qui sont généralement des hommes. Des militants des organisations d'extrême-gauche, de groupes libertaires et de syndicats participent à cette lutte. On trouve par ailleurs des documents de réflexion et des tracts pour des manifestations féministes dans des fonds de syndicats et de groupes politiques ou confessionnels, comme le fonds de la Confédération des syndicats médicaux français d'Ille-et-Vilaine, celui de l'Union départementale CGT d'Ille-et-Vilaine, de la Confédération française démocratique du travail (CFDT) dans les Côtes-d'Armor ou du Cercle Jean XXIII à Nantes ${ }^{11}$.

8 Les tracts sont relativement nombreux mais les images sont rares. Il ne semble pas y avoir eu de photographe comme Catherine Deudon ${ }^{12}$ ou de vidéaste comme Carole Roussopoulos ${ }^{13}$ dans l'Ouest. À la Cinémathèque de Bretagne, deux vidéos réalisées dans les années 1970 témoignent de luttes de femmes. Il s'agit de Clito va bien et de Quand les femmes ont pris la colère. Clito va bien est un film d'environ 34 minutes réalisé en 1979 par le groupe femmes de Quimper et du Planning familial de Brest. Ce film mêle témoignages sur des sujets comme la sexualité ou la ménopause, des scènes théâtralisées (reconstitution d'une consultation gynécologique, sketch sur les règles), des scènes explicatives sur la santé des femmes et de l'auto-observation ${ }^{14}$. Quand les femmes ont pris la colère est un film de Soazig Chappedelaine Vautier et René Vautier, d'une durée de $1 \mathrm{~h} 11$, couleur et sonore, réalisé en 1977. Il documente la lutte des femmes des ouvriers de l'Usine Tréfimétaux de Couëron entre 1975 et 1976. Deux photographies ont été numérisées et sont disponibles sur des bases de données en ligne : la photographie d'une manifestation à Brest en $1977^{15}$ et la photographie d'un enfant qui regarde un graffiti féministe numérisée par le Musée de Bretagne ${ }^{16}$. La question suivante a été posée en entretien aux militantes : «Avez-vous conservé des photographies ou films des luttes féministes dans lesquelles vous étiez impliquées pendant les années 1970 ? ». La réponse la plus fréquente a été celle-ci : « On n'était pas là à prendre des photos de ce qu'on faisait ». J'ai pu toutefois avoir accès à quelques photos de groupes femmes comme celui qui a été à l'origine du café féministe La Marg'elle à Rennes, celui des ateliers bricolage entre femmes auxquels a participé une vendéenne en région parisienne ou de manifestations, comme celle de la fête des Mères à Brest en 1978. Les images de luttes se trouvent plutôt dans la presse quotidienne régionale (Ouest France, le Télégramme, le Courrier de l'Ouest, Presse Océan, Le Maine Libre) et la presse militante locale de gauche (APL-Nantes, Le Chapeau Rond Rouge, Le Canard de Nantes à Brest). 
Les féministes de l'Ouest déposent-elles leurs archives et si oui, où le font-elles ? À l'exception de Ghislaine Ménage ${ }^{17}$ qui a déposé ses archives aux Archives municipales de Rennes, les autres fonds disponibles se trouvent au CAF. Il s'agit des fonds d'AnneMarie Charles (38 AF, Angers), du collectif femmes de Saint-Nazaire (40 AF, SaintNazaire), de Marie-Françoise Gonin, (45 AF, Nantes), de Michèle Grosjean (46 AF, Nantes), de Marie-Madeleine Tallineau (54 AF, Nantes) et d'Anne-Marie Giffo-Levasseur (55 AF, Nantes $\left.{ }^{18}\right)$. Le choix du lieu se fait selon plusieurs critères: connaissance de l'existence du lieu, contact avec un.e membre de l'association Archives du féminisme voire adhésion à l'association, connivence avec un lieu qui rassemble les archives des luttes féministes, personnalité et rayonnement de la présidente de l'association (Christine Bard), inscription dans un réseau d'associations et de personnes qui y ont déjà déposé leurs archives, intégration du CAF dans une structure de recherche qui permet une valorisation des fonds déposés (Grailles 2011 : 173-185). Certaines de ces donatrices sont décédées, mais j'ai pu rencontrer des militantes nantaises qui ont confirmé que leur démarche de don est militante et vise la préservation et la transmission de leur histoire. Elles ont témoigné d'une volonté de s'inscrire individuellement dans un réseau militant et de participer à la transmission d'une aventure collective. Elles ont d'ailleurs également accepté d'être filmées pour enrichir le fonds d'archives audiovisuelles "Témoigner pour le féminisme ». En amont du don ou du dépôt de leurs archives, les militantes opèrent un tri et un classement de cellesci. C'est l'occasion pour certaines d'écrire une chronologie des actions et de restituer des noms. Elles s'assurent ainsi de la transmission d'informations qui peuvent servir aux chercheur.e.s et autres publics, espérant, explique l'une d'entre elles, que des erreurs ne soient pas commises par rapport à la version des faits qu'elles ont établi. Aux prénoms et pseudonymes succèdent donc des identités et un travail d'historicisation des luttes qui rompt avec une habitude prise parmi les groupes femmes de ne pas se nommer et de parler au nom d'un collectif. Il s'agit en effet de témoigner d'une histoire qu'elles pensent peu ou mal connue par les jeunes féministes. D'autre part, c'est une façon d'inscrire la lutte des femmes dans une histoire plus large des mouvements sociaux qui ont marqué cette deuxième moitié du $\mathrm{XX}^{\mathrm{e}}$ siècle. En Bretagne (LoireAtlantique comprise), il est également apparu comme important d'enrichir l'histoire des luttes féministes perçues comme réduite au mouvement parisien. Ces initiatives sont le plus souvent le fait d'anciennes enseignantes.

10 Ces fonds sont peu nombreux au regard de la multitude des groupes féministes actifs en Bretagne et Pays de la Loire durant la décennie 1970. Des militantes ont exprimé leur regret de ne pas avoir accordé d'importance à la matérialité de leurs luttes ou de ne pas avoir eu le réflexe de conserver les tracts, panneaux et autres objets. Les déménagements successifs, la dislocation des groupes et l'éloignement des militantes entre elles ont contribué à cette disparition. Quelques-unes m'ont montré des archives qu'elles conservent comme des souvenirs personnels et m'ont posé des questions : Ces archives ont-elles une valeur, un intérêt historique ? Cela intéressera-t-il ? Quand les transmettre? De mon vivant? Que vont faire mes descendant.e.s de "ce bazar»? Certaines d'entre celles qui se posent ces questions, provoquées par la situation d'enquête, ont franchi le pas de donner le peu d'archives qu'elles possédaient en se disant qu'elles allaient au moins servir à ma recherche. J'ai ainsi en ma possession un tract sur lequel est transcrit une chanson du MLAC de Brest, un numéro de la revue nantaise Dévoilées qui manque au CAF, une affiche du Planning familial de la Roche-surYon et de Saint-Brieuc, une affiche du groupe femmes de Villejean de Rennes dessinée 
et peinte par Andrée Chapalain. D'autres continuent à me parvenir. Une fois ma recherche terminée, ces archives seront déposées au CAF.

\section{Les mots pour dire le genre et la sexualité dans les archives écrites}

11 Travailler sur l'expérience de libération sexuelle des militantes du mouvement des femmes, c'est être confronté.e à une double difficulté. D'abord les archives produites par les militantes elles-mêmes sont rares, on l'a vu. Aussi, les archives des luttes féministes supposent une lecture en creux, une lecture entre les lignes. Elles peuvent aborder des aspects intimes de la vie des militantes, mais souvent à la marge. Les tracts sont l'étendard des revendications liées aux expériences des militantes. Il s'agit toutefois de bribes, de fragments d'expériences décontextualisées, généralisées et transformées par la mise sur la place publique. Je n'ai pas eu accès à des journaux intimes ni à des échanges épistolaires. Alors comment comprendre, par exemple, l'expérience des militantes qui ont eu recours à un avortement? Voici deux exemples de documents issus de fonds d'archives publiques qui répondent en partie à cette question. Une militante qui n'aurait pas pu avorter avant la légalisation de l'IVG en France ou même après, pouvait se rendre dans un pays étranger pour le faire, aidée du MLAC, de Choisir ou du Planning familial. Grâce à la conservation du courrier du British Pregnancy Advisory dans le fonds $56 \mathrm{Z}$ des Archives municipales de Rennes, on connait le contenu de ce que peut-être une lettre d'admission dans un service d'avortement en Grande-Bretagne.

\footnotetext{
Chère Mme R.

Un rendez-vous a été pris pour que vous puissiez voir notre conseiller et nos médecins référents, pour évaluer si votre situation rentre dans la cadre de la loi sur l'avortement de 1967 [Abortion Act 1967] et pour un examen médical, le 21 août à 14 heures. Si nos médecins estiment qu'une interruption de grossesse est justifiée dans le respect de la loi, vous serez alors admise à la maison de soins le 23 août, puisqu'un lit provisoire a été réservé pour vous à cette date. Vous devez prévoir un séjour de vingt-quatre heures à compter de votre admission.

Il est possible d'organiser un hébergement pour la nuit précédant votre admission à la maison de soins pour un cout minime. Veuillez nous faire savoir si vous souhaitez que l'hébergement soit réservé pour vous.

Lors du premier rendez-vous, une commission de 10,00£, comprenant l'examen médical et les tests pour déceler une éventuelle maladie doit être payée en espèces. Avant votre admission à la maison de soins, et à condition que votre grossesse puisse être interrompue par une méthode simple, un supplément de 41,00£ doit être payé en espèces pour couvrir le cout de l'opération et des soins afférents. Aucun autre frais ne sera facturé, à l'exception de la nuitée précédant l'admission.

Nous espérons que le rendez-vous vous conviendra. Veuillez apporter un échantillon d'urine et votre numéro de service national de santé19.
}

Comment cela se passe-t-il pour une militante qui veut avorter à Laval après le vote de la loi Veil ? Le fonds 1772W4 des Archives départementales de la Mayenne est constitué des archives du premier centre d'IVG à Laval, créé en 1976. Les rapports qu'il contient donnent le détail des méthodes utilisées, du matériel, des démarches à effectuer, des techniques médicales et des réactions physiques des personnes. Une femme qui arrive 
pour une IVG dans ce centre doit être à jeun et vessie vide. Elle reçoit une prémédication puis subit l'intervention avec "anesthésie verbale " (forme d'hypnose ou de méditation) qui dure de 5 à 10 minutes environ. «L'intervention est pratiquée sans champ opératoire ni gants stériles, l'opérateur appliquant la technique du “'No Touch" et n'utilisant que du matériel parfaitement stérile ${ }^{20}$. » En cas de spasme du col de l'utérus, elle reçoit une injection de spasmolytique. Elle se repose 1 à 2 heures après l'intervention et lui est remise une ordonnance pour des antibiotiques, une contraception, des conseils et un rapport pour le médecin traitant. Analyser une expérience d'avortement, c'est aussi mettre en lumière toute la logistique et le cout que cela implique. Autour des récits collectés auprès des militantes s'esquissent peu à peu, au fil des recherches, tout un univers législatif et une réalité matérielle souvent occultés ou peu détaillés dans ces récits.

Les revues militantes constituent ma source principale. J'ai travaillé sur deux revues créées par des militantes de groupes femmes dans l'Ouest, la revue Dévoilées et Les Femmes et les femmes d'abord. Dévoilées a été publiée de 1979 à 1983, c'est un « journal fait par des femmes pour des femmes sur la région nantaise » créé par cinq militantes puis neuf dans le dernier numéro. Elle comporte neuf numéros. Chaque numéro est structuré d'une façon unique, avec parfois une thématique centrale ou parfois un patchwork de sujets. Les Femmes et les femmes d'abord est une revue qui comporte quatre numéros, de janvier à décembre 1980. Une vingtaine de femmes originaires de différentes villes contribuent à ces numéros : Tours, Angers, Caen, Poitiers, Orléans, StJean d'Angély (et d'autres villes non précisées). La sexualité n'est pas l'objet principal de ces revues. Elles mettent en lumière le quotidien des femmes, leurs problématiques et revendications.

Interroger la sexualité c'est travailler sur la perception et l'expérience du corps de l'autre et de son propre corps, c'est analyser comment les militantes définissent l'amour, ce sont les expériences partagées de conjugalité, de plaisir mais aussi de violence. C'est aussi comprendre l'utilisation des méthodes contraceptives, le choix de la maternité ou de la non-maternité et le recours à l'IVG. C'est enfin penser le rapport aux normes, tant dans les pratiques que dans les identités ${ }^{21}$. Se dévoiler, pour une contributrice anonyme au numéro 5 de la revue Dévoilées, c'est interroger les mots qui nous définissent :

\footnotetext{
Je suis lesbienne, et oui, ça leur fait drôle ce mot-là, ils en sont gênés pour moi, et quand ils n'osent pas dire que ça les met mal à l'aise, ils se justifient en expliquant qu'on n'a pas besoin de se définir sexuellement que c'est de la provocation. Mais qui provoque? qui définit? qui vous met de force dans la norme ? Vous êtes mariée ? vous êtes célibataire. J'en ai assez de ces pièges à mensonge. Je suis lesbienne et vous avez voulu me voler ce mot-là. Moi aussi quand j'étais petite c'est un mot qui me faisait pleurer parce qu'une fille de ma classe m'avait traitée de "sale lesbienne ». J'aimais d'amour ; j'aimais trop fort, c'est suspect, c'est sale, je devais avoir honte. Seulement j'ai grandi et j'ai retrouvé malgré vous le vrai sens de ce mot, il veut dire Femmes, Amour et Liberté22.
}

15 Les textes qui évoquent la sexualité prennent plusieurs formes: témoignages (correspondance, dissertation, tribune), dossiers thématiques, poèmes/chansons, dessins, présentations d'ouvrages et de productions artistiques, intertextualité. On peut distinguer deux types de témoignages : le témoignage d'une situation dont la personne qui écrit est le sujet principal ou le témoignage d'une situation dont la personne qui 
écrit est le témoin. Voici un exemple d'une militante ${ }^{23}$ du mouvement des femmes, qui en accompagne une autre pour un avortement à l'hôpital St-Jacques à Nantes. Ce témoignage permet de comprendre et d'analyser ce que ces expériences font vivre aux militantes. L'avortement est précédé d'un examen gynécologique et le médecin refuse que la patiente soit accompagnée lors de cet examen. C'est à ce moment-là que la narratrice explique avoir opté pour le silence et la réserve afin de ne pas compromettre le bon déroulement de l'avortement de celle qu'elle accompagne, mais elle vit mal cette situation :
J'ai l'impression d'avoir été flouée. Et n'empêche qu'on a intérêt à le ménager aussi, parce que c'est lui qui a fait l'avort [sic] et qu'il a tout pouvoir pour que ça se passe plutôt bien ou plutôt mal. [...] Et X qui est là sur la table, avec les «bougies » dans l'utérus et qui a mal et le toubib qui pendant ce temps passe son temps à essayer de faire tenir la lampe qui éclaire le champ opératoire. J'en ai marre, je lui propose de tenir la lampe et qu'il finisse son boulot. Pas de réponse, j'ai envie de le claquer. [...] Y'a une boite aux lettres marqué: « vos remarques» dans le couloir. Tout ce qu'on n'a pas pu dire, qu'on avait envie de dire, on le crache sur le papier... un peu dérisoire... mais c'est toujours ça de pris $^{24}$.

La revue devient alors un espace où briser ce silence, à l'image de cette boite aux lettres réservée aux remarques mentionnée à la fin du témoignage. Cette boite aux lettres s'avère être un exutoire en même temps que l'unique espace de parole réservé à celles qui sont muselées pendant la visite médicale. On peut s'interroger sur la petitesse de cet espace en même temps que sur sa finalité. Plusieurs autres textes font écho aux expériences de militantes qui accompagnent des femmes. J'ai alors accès à des fragments d'expériences.

17 J'ai pu remarquer une relative spontanéité dans ces écrits et l'usage d'un langage familier. Toutefois, tout travail d'écriture implique des formes de censure, de transformation du réel, d'adaptation de l'expérience et de la pensée en mots. Dans le numéro $1^{25}$, un texte utilise les mêmes codes que ceux du journal intime. Il est manuscrit alors qu'une grande majorité des textes sont dactylographiés. Il est signé "Catherine » et commence comme ceci : "Sympa aujourd'hui, journée avec Nicole, avec plein de complicité, plein de sentiments, d'impulsions latentes. Marrant, envie de caresser la peau soyeuse, douce, tendre, bulle de chaleur, d'affection, je me sens pleine, bien, je prends toute l'ampleur de mes ailes, ou presque». Elle parle de ses sensations, de ses émotions et mêle la poésie à une dénonciation d'un système qui tend à normaliser et à codifier les comportements sexuels et les identités: «Ils nous construisent un code de vie marginale, un code de libération de la femme, du couple, sexuelle et tout court, on dit "homosexuels", les "artistes", les "féministes", les "marginaux".» Ce texte joue avec les mots tout en proposant de les faire éclater, d'éclater les « je t'aime », les « je désire ». Pour Catherine, ces normes et ces injonctions ne peuvent qu'être vaincues face aux palpitations de la vie, aux puissances inconnues, aux silences et aux regards, aux frémissements de la nature et aux rythmes multiples (ceux des saisons, de la mer, du cosmos).

18 Ce sont dans les poèmes que j'ai accès à des informations sur l'acte sexuel en lui-même, mais de façon très imagée, par métaphore. Les autres textes en évoquent les conséquences (contraception, avortement, violences...) mais pas l'intimité de l'acte. 
Comme si la poésie permettait de tout dire, sans trop non plus accorder d'attention à la forme : les vers sont libres.

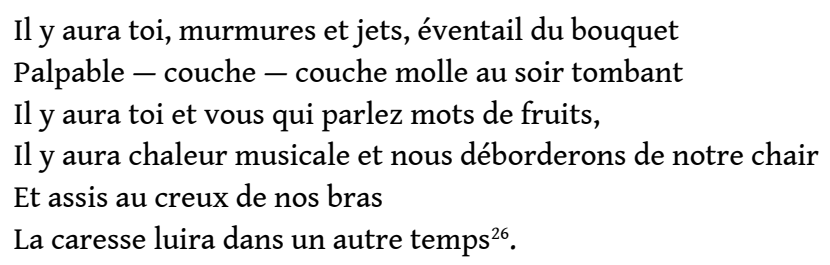

19 Ces poèmes sont, dans une très grande majorité, accompagnés d'une illustration. Les formes abstraites (courbes et volutes), les corps nus de femmes associés au végétal ou à la sorcellerie papillonnent autour des strophes.

Dans l'ensemble des numéros des deux revues, les illustrations sont très diversifiées et aèrent les textes. On retrouve des images issues d'autres revues, des personnages comme Bécassine, des dessins réalisés par les militantes elles-mêmes ou des personnes de leur entourage. Nombreux sont les photographies et collages d'images découpées qui sont en lien avec le thème de la page. La bande dessinée est présente sous forme de vignettes dispersées au fil du texte. Contrairement à d'autres revues féministes, l'amour, la conjugalité, la sexualité ne sont pas représentées à l'exception d'un dessin d'un couple de femmes entrelacées. Ce sont essentiellement des scènes qui illustrent le monde du travail, les tâches ménagères, des manifestations ou des corps de femmes stylisés. Le dessin n'a pas de vocation didactique et dénote une grande part d'humour dans la dénonciation.

Les références à des textes, des chansons, poèmes, revues sont nombreuses. Elles laissent entrevoir les réseaux que tissent les militantes avec d'autres voix, et les échos entre des textes qu'elles citent et leur vie. L'intertextualité, la citation, les recensions d'ouvrage, la bibliographie sont des manières d'aborder des sujets intimes comme les pratiques sexuelles, la masturbation, l'expérience de l'orgasme et la perception du plaisir. Dans le numéro 2 de Dévoilées, une femme restée anonyme fait la recension du livre L'orgasme au féminin de Christine L'Heureux paru en 1979 et réédité en 1982. Elle présente l'ouvrage et fait le lien entre la lecture et son expérience personnelle qu'elle généralise par le «nous » qui désigne les femmes : « Notre culture a toujours enseigné que le sexe de la femme était laid, sale, obscène, même qu'il nous manquait... Et bien, non! Oh! découverte ces dessins nous apprennent la diversité du sexe féminin, aux formes étonnamment belles dans leur différence. Betty Dodson nous dévoile la variété à l'infini, jamais le même aspect, une position du clitoris différente d'une femme à l'autre ». Cet exemple est une forme de témoignage sur la perception du sexe des femmes. Il constitue un écho aux témoignages oraux recueillis pendant la thèse. Si la source écrite, bien que rare, s'avère finalement une ressource importante et complémentaire pour comprendre les expériences personnelles, le récit constitue toutefois la source principale qui replace le témoin dans son parcours de vie et son individualité. 


\section{Les archives orales : enrichir le rien et rendre visibles des parcours de femmes}

22 lacunaire, reconstruite... Pour reprendre la question que pose Florence Descamps,
spécialiste de l'histoire orale, «que puis-je attendre des sources orales?» (Descamps 2006 : 41). Elle y répond en ces termes «Elles rendent possible le croisement des itinéraires personnels et collectifs » (Descamps 2006 : 220). Comprendre l'expérience de libération sexuelle des militantes c'est effectuer des va-et-vient entre les évènements (Mai 68, Mouvement des femmes, autres engagements militants, réformes législatives, etc.) et les trajectoires personnelles des militantes. Si l'histoire s'intéresse aux évènements, l'histoire orale pose une autre question: qu'est-ce que ces évènements signifient? (Portelli 2016: 22). La source orale est au cœur de cette thèse sur l'expérience subjective d'une traversée politique des années 1970.

J'étais prise, en début de thèse, dans ce qui m'apparaissait comme une contradiction: garantir l'anonymat et accéder aux informations que j'estimais les plus intimes d'une personne; rendre visibles les militantes par la constitution d'un fonds d'archives consultable. La solution trouvée a été de réaliser les entretiens en deux temps avec deux techniques. La première partie de l'entretien était filmée et non directive. L'objectif était de constituer une archive orale qui viendrait enrichir le fonds "Témoigner pour le féminisme» de l'association Archives du féminisme ${ }^{27}$. Un travail collectif ${ }^{28}$ s'est élaboré au fil des mois pour penser une méthodologie de collecte et écrire un contrat de témoignage qui sécuriserait la démarche et définirait les conditions d'utilisation et d'accès des témoignages. L'aspect non directif de cet entretien permettait aux militantes de se dire dans les limites de ce qu'elles concevaient et de développer leur parcours et exposer leurs autres engagements éventuels. La deuxième partie de l'entretien était anonyme, enregistrée sur dictaphone et semi-directive. Elle allait plus directement servir mon travail de thèse et me faire accéder à la vie sexuelle et affective des militantes. J'ai réalisé 37 entretiens filmés et 38 entretiens anonymes pour un total de 46 personnes rencontrées. La durée moyenne des entretiens filmés a été d' $1 \mathrm{~h} 30,40$ minutes pour le plus court et $2 \mathrm{~h} 30$ pour le plus long. Les entretiens anonymes, quant à eux, ont duré entre 1 et 6 heures environ. La militante la plus âgée est née en 1931 et la plus jeune en 1955. Elles sont $30 \%$ à être nées en Bretagne, $37.5 \%$ en Pays de la Loire et $32.5 \%$ à être nées dans une autre région. $73 \%$ d'entre elles ont milité dans un groupe femmes non mixte, au MLAC ou à Choisir, $20 \%$ ont milité exclusivement au Planning familial, 7 \% à l'Union des femmes françaises (aujourd'hui Femmes Solidaires) ou au CIDF (Centre d'Information pour les Droits des Femmes et des Familles) et à la Délégation régionale aux droits des femmes ${ }^{29}$. Les 37 personnes qui ont été filmées ne sont pas nécessairement les 38 personnes qui ont témoigné de façon anonyme. Les deux types d'entretiens n'ont pas été proposés systématiquement à chaque personne, soit parce que les personnes n'entraient pas dans le cadre précis de mon travail de thèse, soit par manque de disponibilité de la personne ou de temps le jour de l'entrevue. L'entretien filmé n'a pas été accepté non plus par toutes les personnes quand celui-ci leur était proposé. Une condition a été posée par certaines, témoigner si d'autres militantes de leur groupe témoignent. Elles l'ont justifié par un souci de représentativité et de non-prise de pouvoir par une visibilité que les autres n'auraient pas. Elles étaient, en effet, conscientes que sortir de

GLAD!, 11 | 2021 
l'anonymat pour devenir un sujet historique et évoquer une expérience collective constituait une prise de pouvoir susceptible de déformer les équilibres militants qui avaient existé et bouleverser les relations encore actuelles. Pour d'autres, celles qui avaient davantage conscientisé le fait d'avoir exercé une influence sur une dynamique militante (voire politique) locale, témoigner était une évidence.

La captation vidéo était proposée en début de rencontre d'abord parce qu'elle constituait, a priori, la phase la plus angoissante de la rencontre et parce qu'elle était la partie la plus techniquement difficile. Elle permettait également d'ouvrir le témoignage avant de cibler les questions et de ne pas avoir à revenir au parcours général de la personne si elle avait d'abord témoigné hors enregistrement. La militante se retrouve face à un dispositif qu'elle ne connait pas (sauf quelques exceptions, les militantes qui ont eu des mandats politiques notamment). Quelle image va-t-elle renvoyer d'ellemême et de ses engagements ? À quelles fins ses propos vont-ils être transmis, utilisés ? Saura-t-elle s'exprimer clairement, de façon cohérente? Comment va-t-on percevoir l'environnement qui sera visible (les entretiens se font au domicile des personnes)? Est-ce que ses propos seront intéressants? Vont-ils se retrouver sur internet? Les questions que peuvent se poser les militantes ont trouvé leur réponse dans le contrat de témoignage et ensuite par la relation d'échange qui s'est établie entre elles et moi. Trois questions revenaient régulièrement au cours de l'entretien anonyme. Si le contrat de témoignage garantissait l'anonymat, des questions subsistaient sur l'utilisation d'extraits. Va-t-on me reconnaitre même avec un prénom différent? Jusqu'où allezvous aller? Que se passe-t-il pour les personnes que je cite? Afin de les rassurer, j'évoquais la manière dont j'allais procéder pour utiliser des extraits de témoignage. Je donnais aussi le nombre d'entretiens réalisés en leur expliquant que ces récits seraient mélangés et pas tous systématiquement utilisés pour chaque thématique. Enfin, je conseillais aux militantes quelques ouvrages sur des sujets proches de ma thèse pour montrer des manières d'écrire sur la sexualitée ${ }^{30}$.

« Ne rien avoir à dire », «ne pas être représentative ", « ne pas être légitime », « ne pas être intéressante ", "ne pas savoir dire»: voici diverses réactions entendues avant l'allumage de la caméra. Cela est nettement moins le cas quand l'entretien est réalisé dans un cadre anonyme et non filmé. Cette hésitation n'apparait pas, ou à la marge, chez celles qui ont eu un mandat électoral, une responsabilité importante dans un parti politique, ou qui ont déjà été en contact avec un.e journaliste, chercheur.e, etc. Elles ont déjà une réflexivité sur leur prise de parole. Une grande majorité de personnes interviewées témoignent sans difficulté. Celles qui ont le plus besoin de préparation (par un dialogue rassurant avec l'enquêtrice, par la préparation de notes ou de documents) sont généralement les plus âgées (+ de 80 ans) et celles qui manifestent de hautes exigences de présentation de soi (posture corporelle, apparence physique, diction, clarté et précision du propos, rapport au corps vieillissant). Les données relatives aux catégories socio-professionnelles ne donnent pas de résultats significatifs pour comprendre ces différences. Après montage, chaque témoignage fait l'objet d'un visionnage par la militante pour validation du contenu. Ces témoignages sont spontanés, rarement préparés. Il m’a été parfois demandé de couper certains passages. C'est après visionnage que certaines disent que c'est intéressant et que cela fait sens. Ce visionnage peut s'avérer difficile car il renvoie une image de soi qui peut ne pas être conforme à ce qu'on imagine de sa prise de parole, de son physique ou de ses habitudes 
de langage, ce que des militantes appellent « les défauts ». Cela peut être ennuyeux car leur histoire, elles la connaissent déjà me disent-elles.

À chaque entretien se pose la question des enjeux éventuellement vécus par le témoin et de ses attentes ${ }^{31}$ : le témoin est-il en représentation ? Quel intérêt accorde-t-il à cette recherche et pourquoi ? La personne a-t-elle témoigné dans d'autres cadres, pour d'autres projets? Quel rapport entretient-elle avec son passé? Manifeste-t-elle des attentes particulières? Enfin, la question de l'autocensure doit se poser, elle rejoint le point précédemment évoqué, mais peut être perceptible à l'analyse des entretiens. Comment la déceler? Par les expressions du visage, les gestes, les moments de pause, les retours en arrière, les reformulations, les hésitations, le ton de la voix, les blocages, les réponses à côté du sujet, les silences. Un des facteurs qui favorise une forme d'autocensure chez les témoins, c'est la perception qu'elles et ils ont éventuellement du positionnement politique, idéologique, intellectuel de la personne qui recueille le témoignage. Ma démarche pour l'association Archives du féminisme, née de mon travail de terrain pour la thèse, m'inscrivait dans une position qu'analyse Isabelle Clair, celle de "faire du terrain en féministe» (Clair 2016: 66-83). Mon héritage féministe s'est d'ailleurs traduit par des questionnements sur le rapport de domination qu'implique une situation d'enquête, le fait de transformer des expériences en objet d'étude, le travail de contextualisation d'expériences personnelles et de déconstruction opérée par le processus d'écriture, le fait de rendre invisible une partie de celles qui ont constitué le Mouvement des femmes à l'Ouest parce qu'elles n'ont pas été interrogées, le recours à l'anonymat qui implique une forme de généralisation et d'invisibilité. Ces réflexions ont amené à des pratiques qui ont été un approfondissement d'une méthodologie s'inscrivant à l'intersection de la sociologie et de l'histoire. J'ai, dans un premier temps, analysé ma position en tant que femme, lesbienne, féministe par rapport à un sujet sur le féminisme. J'ai ainsi explicité, pour moi, les connivences éventuelles, ou au contraire les divergences intellectuelles et idéologiques avec mes enquêtées. "Enquêtée » est d'ailleurs un terme que je n'utilise pas sur le terrain et de façon marginale dans l'écriture, je lui préfère celui de «militante». Seulement deux personnes m'ont interrogée par rapport à mes positionnements sur des sujets qui clivent les féministes : prostitution, voile, GPA. Ces questions ont été posées à la fin de l'entretien, au moment où les militantes me paraissaient plus à l'aise et manifestaient un intérêt et une légitimité à me poser des questions. Celles-ci n'ont pas compromis l'entretien qui était terminé. J'ai accordé du temps à la discussion sur le consentement des militantes par rapport au processus de l'entretien et notamment le dispositif de la caméra ainsi que la teneur des questions posées. Ensuite, je n'ai cherché à exclure aucune personne parmi celles qui ont souhaité spontanément témoigner, leur accordant le crédit du fait de se sentir concernée par le sujet quand bien même certaines d'entre elles étaient à la marge par rapport au cadre qui était le mien, celui du Mouvement des femmes dans les années $1970^{32}$. Une exception a été faite avec une femme n'ayant eu aucun engagement féministe et qui souhaitait se servir de mon travail comme tribune pour ses positions. Une large place a été accordée à la restitution de mon travail pendant tout le processus de la thèse. Deux films ${ }^{33}$ ont été réalisés à partir des témoignages filmés et ont fait l'objet de plusieurs projections. Un carnet Hypothèses ${ }^{34}$ permet aux militantes de suivre mes découvertes en archives sur les associations féministes et groupes femmes locaux ${ }^{35}$. Les militantes n'ont pas seulement été un objet d'étude, elles ont été mobilisées dans le processus de valorisation. Les projections de films s'organisent grâce à la mobilisation de leurs propres réseaux. 
Après trois ans de recherche, ce «rien » à Brest s'est transformé en blog de recherche, des films et bientôt une thèse. Ce «rien» est devenu également tout un réseau de militantes prêtes à soutenir une démarche et qui ont témoigné et c'est ce qui me permet aujourd'hui de briser un silence "provincial». Les craintes du rien apparaissent désormais comme infondées pour qui sait tendre l'oreille et ouvrir les yeux. Le rien n'est pas rien et il attend d'être mis en lumière dans les bases de données des centres d'archives, dans les façons de lire, de regarder et de décrire et par la valorisation scientifique aussi bien que militante.

\section{BIBLIOGRAPHIE}

ARTIERES, Philippe. 2009. « Histoires d'archives » Revue historique 649 (1) : 119-126.

BARD, Christine, METZ, Annie \& NEVEU, Valérie (éd.). 2006. Guide des sources de l'histoire du féminisme. Rennes : Presses Universitaires de Rennes.

BLUM, Françoise (éd.). 2017. Genre de l'archive : constitution et transmission des mémoires militantes. Paris : Codhos.

CLAIR, Isabelle. 2016. « Faire du terrain en féministe » Actes de la recherche en sciences sociales [En ligne], 213 (3), consulté le 23 septembre 2021. URL : https://www.cairn.info/revue-actes-de-larecherche-en-sciences-sociales-2016-3-page-66.htm (https://doi.org/10.3917/arss.213.0066).

DESCAMPS, Florence. 2006. Les sources orales et l'histoire, Rosny-sous-bois : Bréal.

GRAILLES, Bénédicte. 2011. «Collecter et rendre visible les archives du féminisme : une action en réseaux » La Gazette des archives [En ligne]. 221 (1), consulté le 23 juin 2021. URL : https:// www.persee.fr/doc/gazar_0016-5522_2011_num_221_1_4783 (https://doi.org/10.3406/gazar. 2011.4783).

PERROT, Michelle. 2008. Mon histoire des femmes. Paris : Seuil.

PORTELLI, Alessandro. 2016 [2009]. The Oral History Reader. London : Routledge.

REBREYEND, Anne-Claire. 2008. Intimités amoureuses : France, 1920-1975. Toulouse : Presses Universitaires du Mirail.

\section{NOTES}

1. Archives du féminisme est une association qui a pour objectifs de collecter les archives privées des militant.e.s et des associations féministes ainsi que les archives concernant la cause des femmes; les sauvegarder, les classer, les inventorier et les mettre à la disposition de la recherche; organiser des colloques, des journées d'études, des expositions destinés à faire connaitre les travaux menés dans ce domaine de la recherche historique ; et plus généralement, prendre toute initiative pour faire connaitre et valoriser les fonds d'archives féministes. 
2. Ce colloque a eu lieu du 26 au 28 mars 2018 à l'Université d'Angers et s'est déroulé pendant le Mois du Genre. Les actes du colloque sous la direction de Christine BARD, Pauline BOIVINEAU, Marion CHARPENEL, Bénédicte GRAILLES et Audrey LASSERRE sont à paraitre aux PUR.

3. Rapport « Grande collecte archives du féminisme » Mission Françoise Thébaud, 2017.

4. Une version en ligne se trouve sur le site de l'association Archives du féminisme.

5. La bibliothèque Marguerite Durand a été fondée en 1932 grâce au don des collections de la journaliste féministe Marguerite Durand à la Ville de Paris.

6. Le CAF été fondé en 2000, par convention entre l'Université d'Angers et l'association Archives du féminisme. Il conserve, en 2021, 45 fonds féministes personnels et 33 fonds féministes associatifs.

7. Tous les centres d'archives de Bretagne et des Pays de la Loire n'ont pas été visités, soit parce que les inventaires et retours des archivistes ne donnaient rien, soit faute d'accès à cause des mesures de confinement liées à la pandémie de Covid-19. Les archives du Mans, inaccessibles pendant plusieurs mois, m'ont envoyé des copies de certains documents d'archives.

8. Le mot «féminisme » a donné un résultat sur le catalogue des AD de l'Ille-et-Vilaine.

9. Une militante d'un groupe femmes nantais m'a parlé de Marie-Françoise le Pichon et de son implication dans l'AG pour l'avortement.

10. Un inventaire détaillé du fonds d'Alain Chénard ainsi qu'une bibliographie est disponible sur le site des $\mathrm{AD}$ de la Loire-Atlantique.

11. Fonds de la Confédération des syndicats médicaux français d'Ille-et-Vilaine (AD Ille-etVilaine, 99 j 165), Fonds de l'Union départementale CGT d'Ille-et-Vilaine (AD Ille-et-Vilaine, $210 \mathrm{~J}$ 79 et 35), Fonds de la Confédération française du travail (CFDT) (AD des Cotes d'Armor, $158 \mathrm{~J}$ 66), Fonds du Cercle Jean XXIII (AD de Loire-Atlantique, $252 \mathrm{~J} 22$ et 49).

12. Catherine Deudon est une photographe et militante féministe qui a photographié de nombreuses militantes et mobilisations du Mouvement de libération des femmes. Voir DEUDON, Catherine. 2003. Un mouvement à soi :

images du mouvement des femmes 1970-2001, Paris : Syllepse.

13. Réalisatrice et militante féministe, Carole Roussopoulos a participé à la création du collectif de distribution Mon ๔Eil, de l'association Les Insoumuses et du Centre audiovisuel Simone de Beauvoir. Voir ROUSSOPOULOS, Carole, 2010. Caméra militante. Luttes de libération des années 1970, Genève : Métis Presses.

14. Je signale ici le film de Marie Hélia, Les Chevalières de la table-ronde, réalisé en 2013 et produit par Paris-Brest Productions, Fée Clochette. Ce long-métrage offre des témoignages de militantes du Planning familial de Quimper, Quimperlé, Brest et du MLAC de Concarneau.

15. Photographie d'une protestation en faveur de l'avortement dans les rues de Brest, auteurice inconnu.e, 1977, archives municipales de Brest, fonds 2 FI04527.

16. Photographie anonyme, noir et blanc, Un enfant regarde un grand graffiti représentant une femme enceinte et légendé: «c'est pas drôle toute l'année à force d'avoir des enfants!!!! Avortement et contraception libres et gratuits. », vers 1975, Musée de Bretagne.

17. Ghislaine Ménage est actuellement impliquée dans l'association Histoire du féminisme à Rennes, qui a pour objet d'explorer, promouvoir et diffuser l'histoire locale des luttes pour les droits des femmes et des vécus individuels qui croisent l'histoire collective des grands combats féministes.

18. Le fonds de l'Espace Simone de Beauvoir conservé au CAF n'est pas encore classé et n'est donc pas accessible.

19. Courrier du British Pregnancy Advisory Service pour un avortement, issu du fonds $56 Z 1$ à 12 des Archives municipales de Rennes. Traduit de l'anglais par Marine Gilis.

20. Dr Hélène Scholl, Dr Marc Valin, «Bilan d'un an de fonctionnement du Centre d'I.V.G. de l'hôpital de Laval (Mayenne) », 1977, AD de la Mayenne, fonds 1772W4. 
21. Des thèses récemment soutenues ou en cours portent sur ces différentes thématiques. Liste non exhaustive : CHARPENEL, Marion. 2014. «Le privé est politique!» Sociologie des mémoires féministes en France. Thèse de doctorat, IEP de Paris, ; DEIBERT-TURNER, Star. 2013. The Golden Speculum: A History of the Vancouver Women's Health Collective, 1970-1983. Thèse de doctorat, Simon Fraser University, Burnaby, Colombie-Britannique ; DEL RIO, Chelsea Nicole. 2016. "That Women Could Matter": Building Lesbian Feminism in California, 1955-1982. Thèse de doctorat, University of Michigan ; ELOIT, Ilana. 2018. Lesbian Trouble: Feminism, Heterosexuality and the French Nation (19701981). Thèse de doctorat, The London School of Economics and Political Science; GURUN, Anna. 2015. Second-wave feminist approaches to sexuality in britain and france, c.1970-c1983. Thèse de doctorat, University of Dundee, Scotland, United Kingdom; JACQUET, Catherine Olga. 2012. Responding to Rape: Contesting the Meanings of Sexual Violence in the United States, 1950-1980, Thèse DE doctorat, University of Illinois at Chicago; MASCLET, Camille. 2017. Sociologie des féministes des années 1970 Analyse localisée, incidences biographiques et transmission familiale d'un engagement pour la cause des femmes en France, Thèse de doctorat, Université Paris 8 et Université de Lausanne ; MATHIEU, Marie. 2016. Derrière l'avortement, les cadres sociaux de l'autonomie des femmes. Refus de maternité, sexualités et vies des femmes sous contrôle. Une comparaison France-Québec, Thèse de doctorat, Université Paris 8 et Université du Québec à Montréal ; RUAULT, Lucile. 2017. Le spéculum, la canule et le miroir. Les MLAC et mobilisations de santé des femmes, entre appropriation féministe et propriété médicale de l'avortement (France, 1972-1984), Thèse de doctorat, Université Lille 2 ; ТНОмÉ, Cécile. 2019. La sexualité aux temps de la contraception. Genre, désir et plaisir dans les rapports hétérosexuels (France, années 1960 - années 2010), Thèse de doctorat, EHESS.

22. Anonyme. 1981. «Je suis lesbienne » Dévoilées 5.

23. Le témoignage est anonyme.

24. Anonyme. 1979. «Visite avant l'avortement » Dévoilées 1.

25. Catherine. 1979. « Autres découvertes... » Dévoilées 1.

26. Odile. 1980. « Il y aura toi... » Les femmes et les femmes d'abord $1: 26$.

27. GILIS, Marine \& GERARDIN-LAVERGE, Mona. 2020. « La commission audiovisuelle d'Archives du féminisme : la dynamique est lancée ! ». Bulletin d'Archives du féminisme 28.

28. La commission audiovisuelle d'Archives du féminisme est composée, en 2021, de 7 membres : Chrystel Grosso, Lucy Halliday, Marie Videbien, Marie-Claude Caillaud, Marine Huguet, Mona Gérardin-Laverge (qui a mené en même temps que moi une collecte d'archives orales) et moimême.

29. Les $7 \%$ sont toutes originaires de la Mayenne.

30. Je conseillais notamment la lecture de l'ouvrage suivant: REBREYEND, Anne-Claire. 2008. Intimités amoureuses : France, 1920-1975. Toulouse : PUM.

31. En plus des ouvrages méthodologiques, articles et ouvrages collectifs sur le thème de l'entretien dans les sciences humaines et sociales, nombreux sont les ouvrages sur la sexualité, l'amour, l'histoire des femmes et du genre qui abordent ces questions, souvent en introduction. En outre, les discussions lors des réunions de la commission audiovisuelle d'Archives du féminisme, les séances organisées par l'association EFiGiES-Ouest, les séminaires, colloques et rencontres doctorales ont constitué des moments d'échanges centraux dans ma réflexivité quant à ma démarche de constitution d'un fonds d'archives orales.

32. Cela a été possible parce que j'ai eu très peu de témoignages proposés spontanément.

33. Un film amateur a été réalisé sur le féminisme en Bretagne dans les années 1970 (GILIS, Marine. 2020. Luttes féministes en Bretagne dans les années 1970) et un autre sur le même sujet en Pays de la Loire (GILIS, Marine. 2021. Luttes féministes en Pays de la Loire dans les années 1970).

34. Un carnet Hypothèses est un carnet hébergé en ligne par la plateforme Hypothèses.org, réservée aux personnes qui ont un rattachement universitaire.

35. Carnet Hypothèses «La recherche comme une aventure »: https:// laventure.hypotheses.org/. 


\section{RÉSUMÉS}

Cet article présente le travail d'exploration des archives écrites et de constitution d'un fonds d'archives orales à partir du sujet de thèse suivant : L'expérience de libération sexuelle des militantes du Mouvement des femmes en Bretagne et Pays de la Loire (1968-1981). Comment trouver et exploiter les archives pour une étude portant sur la sexualité ? Que faire en cas d'absence de ces archives? Comment articuler recherche, constitution et valorisation d'archives orales ? Les archives écrites relatives aux luttes féministes dans les années 1970 dans l'ouest de la France métropolitaine sont ici analysées. Les sources orales sont évoquées à travers un travail de collecte mené dans le cadre d'une thèse et de la commission audiovisuelle de l'association Archives du féminisme.

This article presents the exploration of written archives and the constitution of an oral archive based on the following thesis topic: The experience of sexual liberation of the activists in the Women's Movement in Brittany and Pays de la Loire (1968-1981). How do we find and use archives for a study on sexuality? What should be done in the absence of these archives? How can we link research with the creation and use of oral archives? The written archives relating to feminist struggles in the 1970s in western France are analyzed here. The oral sources referred to come from a collection constituted within the framework of a thesis and of the audiovisual commission of the association Archives du féminisme.

\section{INDEX}

Thèmes : Recherches

Keywords : archives, feminism, lesbianism, Bretagne, Pays de la Loire

Mots-clés : archives, féminisme, lesbianisme, Bretagne, Pays de la Loire

\section{AUTEUR}

\section{MARINE GILIS}

Marine Gilis est doctorante en histoire à l'Université d'Angers, rattachée au laboratoire TEMOS (UMR 9016 CNRS). Elle travaille sur l'expérience de libération sexuelle des militantes du Mouvement des femmes en Bretagne et Pays de la Loire (1970-1981). Elle est également membre de la commission audio-visuelle de l'association Archives du féminisme. C'est avec cette double casquette qu'elle collecte des témoignages et réalise des films pour les partager au grand public. 